\title{
Study of power network line damage identification based on image feature analysis
}

\author{
LI Hongzhao ${ }^{a}$, YANG Fumin ${ }^{b}$
}

Beijing Polytechnic, \#9 Liang Shui He First Street, Yizhuang Economic Development Zone, Daxing, Beijing, China Postal Code: 100176

alihongzhao198@163.com, byangfumin197@163.com

Keywords: power network, image feature, line damage, identification

\begin{abstract}
This paper proposed a power network line damage identification method based on image feature analysis. By extracting and analyzing outline features, shape features and statistical characteristics of electric power network images, the classification results of all the sub images of all the to-be-matched vein images are fused, whether this image matches the power network line breakage characteristics is judged, so as to realize the power network lines' damage identification, solve the problem of the traditional line identification method as a result of the kinds of line breakage it can analyze is limited and it can't complete lines' damage identification problem effectively.The simulation results show that the proposed method has high identification accuracy.
\end{abstract}

\section{Introduction}

In recent years, with the rapid development of power network, relevant management departments need to regularly check the usage of power network lines, to take the corresponding maintenance countermeasures for broken lines[1, 2]. How to quickly make investigation on the whole power network lines and identify problem areas without affecting normal network communication, has gotten more and more attention, and become the important challenge for the relevant scholars [3-5].

In the process of damage identification for the circuit of the electric power network, it is prone to have the problem of a great variety of line breakage, this led to the traditional electric power network identification method based on wavelet neural network algorithm, because of limited kinds of line breakage it can analyze, cannot effectively complete power lines' damage identification.

This paper puts forward a kind of power network line damage identification method based on image feature analysis, preliminary processing was done on the image of power network line damage through the contour features. Further description is done on the characteristics of power network line breakage through torque and contour description. For the image of digital electric power network lines, use the form of the double sum to replace integrals, conduct statistical analysis on the image gray value, and use it as the statistical characteristics of the image. After training the sample to finish studying according to the known category, conduct study classification on all the test samples, obtain the correct classification rate of sub images at different positions, and calculate the weight of each image when matching. Conduct fusion on the classification result of all the sub images of all to-be-matched vein images, judge whether the image matches with electric power network line breakage characteristics, so as to realize the power lines' damage identification. The simulation results show that the proposed method has high identification accuracy.

\section{Identification of power network line's damage based on the wavelet neural network}

At present, both in China and abroad, the study of wavelet neural network usually tends to tackle bottleneck problems such as "redundancy" of wavelet neural network and parameters initialization. This paper identifies the breakage of the electric power network line based on the discrete wavelet neural network.

Learning algorithm of wavelet neural network.

$h(t)=\cos (1.75 t) \exp \left(-t^{2} / 2\right)$ is Morlet function, which is used for the wavelet basis function. 
Assume $E=\frac{1}{2} \sum_{t=1}^{T}\left(d_{n}-f_{n}\right)^{2}, \quad d_{n}$ is the expected classification output of the signal $x_{n}(t)$, $t^{\prime}=\left(t-b_{j}\right) / a_{i}, \quad \sigma^{\prime}=\frac{\partial \sigma(u)}{\partial(u)}=\sigma(u)[1-\sigma(u)]$, the gradient of $E$ to parameters $\omega_{i j}, a_{i}$, and $b_{j}$ can be described respectively as:

$$
\begin{aligned}
& \frac{\partial E}{\partial \omega_{i j}}=\sum_{n=1}^{N} \sum_{t=1}^{T}\left(d_{n}-f_{n}\right) \sigma^{\prime}\left(u_{n}\right) x_{n}(t) \\
& \cos \left(1.75 t^{\prime}\right) \circ \exp \left(\frac{-t^{\prime 2}}{2}\right) \\
& \frac{\partial E}{\partial \omega_{i j}}=-\sum_{n=1}^{N} \sum_{t=1}^{T}\left(d_{n}-f_{n}\right) \sigma^{\prime}\left(u_{n}\right) x_{n}(t) \omega_{i j} \\
& {\left[1.75 \sin \left(1.75 t^{\prime}\right) \circ \exp \left(\frac{-t^{\prime 2}}{2}\right) \circ \frac{1}{a_{i}}+\right.} \\
& \left.\cos \left(1.75 t^{\prime}\right) \circ \exp \left(\frac{-t^{\prime 2}}{2}\right) \circ \frac{t^{\prime}}{a_{i}}\right] \\
& \frac{\partial E}{\partial a_{j}}=-\sum_{n=1}^{N} \sum_{t=1}^{T}\left(d_{n}-f_{n}\right) \sigma^{\prime}\left(u_{n}\right) x_{n}(t) \omega_{i j} \\
& {\left[1.75 \sin \left(1.75 t^{\prime}\right) \circ \exp \left(\frac{-t^{\prime 2}}{2}\right) \circ \frac{t^{\prime}}{a_{i}}+\right.} \\
& \left.\cos \left(1.75 t^{\prime}\right) \circ \exp \left(\frac{-t^{\prime 2}}{2}\right) \circ \frac{t^{\prime}}{a_{i}}\right]
\end{aligned}
$$

Identification of power network lines' breakage based on the wavelet neural network. This paper divides breakage of power network lines into three categories: longitudinal crack damage, transverse crack damage and good lines. Details of its processing process is: first, value filter processing is done on the electric power network lines' image, then, it is divided into several blocks of the same size, conduct regression analysis according to the local average in blocks to get the local threshold, completes binarization processing through local threshold. Finally make sub-block 0,1 stereotyped according to the percentage of damaged pixels in sub-blocks, assign 0 to the sub-block whose percentage of damaged pixels does not exceed 1\%, assign 1 for the other occasion.

After binarization processing on the sub-blocks of the power network lines, execute a convolution on two density factors $3 \times 3$ and $2 \times 2$, with the corresponding pavement image, $S_{3}$ and $S_{2}$ are obtained:

$\mathrm{S}_{3}=\{$ Convolution on $3 \times 3$ density factor and lines' image $\}$ (4)

$\mathrm{S}_{2}=\{$ Convolution on $2 \times 2$ density factor and lines' image $\}$ (5)

Assuming that the total number of the sub-block image "1" of the original power grid lines is $S_{0}$, calculate the characteristics:

$$
\left\{\begin{array}{l}
F 1=\left(S_{3}-S_{0}\right) / S_{0} \\
F 1=\left(S_{2}-S_{0}\right) / S_{0}
\end{array}\right.
$$

Use $S_{0} 、 F 1$ and $F 2$ as the electric power network's line damage characteristics. Input neural network, the output of the neural network is the electricity network's line damage's identification results.

In the process of power network lines' damage identification, it is prone to have the problem of the variety of line breakage is great, which leads to the above power network line identification 
methods' analysis based on wavelet neural network algorithm, because of the limited variety of line breakage it can analyze, cannot effectively complete power lines' damage identification. Therefore, this paper put forward a kind of power network lines' damage identification method based on image feature analysis.

\section{Power network lines' breakage identification based on image feature analysis}

Electric power network lines' breakage features' extraction. Feature extraction is actually a kind of transformation. Feature extraction is the key to damage identification for the power network, is the guarantee of the success rate of target recognition and identification efficiency.

(1)Contour feature extraction. Contour feature is a significant feature of target images, preliminary treatment is done usually by contour features of power network lines' broken images.

Assume that the contour edge coordinates are $p_{i}=\left[x_{i}, y_{i}\right]^{T}$, the contour is $\mathrm{N}$ random vectors' collection $P, P=\left\{p_{i}\right\}, i=1,2, \cdots, N$.

Average vector, i.e., the centroid coordinates of power lines can be described as:

$\mu=\frac{1}{N} \sum_{i=1}^{N} p_{i}$

The average radius $\mu_{r}$ can be described as:

$\mu_{r}=\frac{1}{N} \sum_{i=1}^{N}\left|p_{i}-\mu\right|$

The covariance matrix $C$ can be described as:

$C=\frac{1}{N} \sum_{i=1}^{N}\left(p_{i}-\mu\right)\left(p_{i}-\mu\right)^{T}$

Assume $C=\left[\begin{array}{ll}C_{x x} & C_{x y} \\ C_{y x} & C_{y y}\end{array}\right]$, the spindle rate can be described as:

$\operatorname{prax}=\frac{C_{x x}+C_{y y}-\sqrt{\left(C_{x x}+C_{y y}\right)^{2}-4\left(C_{x x} C_{y y}-C_{x x}+C_{x y}^{2}\right)}}{C_{x x}+C_{y y}+\sqrt{\left(C_{x x}+C_{y y}\right)^{2}-4\left(C_{x x} C_{y y}-C_{x x}+C_{x y}^{2}\right)}}(10)$

The spindle rate is used to describe the length-width ratio of electric power network lines.

The elliptical variance can be described as:

$\sigma_{e}=\frac{1}{N \sigma_{r e}} \sqrt{\left(p_{i}-\mu\right)^{T} C^{-1}\left(\left(p_{i}-\mu\right)-\mu_{r e}\right)^{2}}$

of which,

$\mu_{r e}=\frac{1}{N} \sum_{i=1}^{N} \sqrt{\left(p_{i}-\mu\right)^{T} C^{-1}\left(p_{i}-\mu\right)}$

The elliptical variance describes the level of similarity between the object's shape and the ellipse with the spindle axis as its axis, the smaller $\sigma_{e}$, its shape is more similar to the oval shape. Perimeter square area ratio is Disp $=S^{2} / \mathrm{A}$.

(2) Image shape feature. The characteristics of power network line breakage is further described through torque and outline description. The torque characteristics is a term used to describe a statistical average, and the two-dimensional torque will be used for shape recognition. For a two-dimensional function $f(x, y)$ of a continuous image, its $(\mathrm{p}+\mathrm{q})$-order moment can be described as the following Riemann integral form:

$m_{p, q}=\int_{-\infty}^{\infty} \int_{-\infty}^{\infty} x^{p} y^{q} f(x, y) d x d y$

Of which, $p, q=0,1,2, \cdots$. According to the principle of uniqueness, if $f(x, y)$ is piecewise 
continuous, and has non-zero values on the $x y$ plane domain, then all order moments exist, at the same time, torque sequence $\left\{m_{p, q}\right\}$ will be determined by $f(x, y)$. Otherwise, $\left\{m_{p, q}\right\}$ solely determines $f(x, y)$. Conduct normalized processing on the torque characteristics, central moment of image $f(x, y)$ can be obtained:

$$
\begin{aligned}
& u_{p, q}=\int_{-\infty}^{\infty} \int_{-\infty}^{\infty}\left(x-x_{c}\right)^{p}\left(y-y_{c}\right)^{q} f(x, y) d x d y \\
& x_{c}=\frac{m_{1,0}}{m_{0,0}}, y_{c}=\frac{m_{0,1}}{m_{0,0}}, m_{1,0}=\int_{-\infty}^{\infty} \int_{-\infty}^{\infty} x f(x, y) d x d y \\
& m_{0,0}=\int_{-\infty}^{\infty} \int_{-\infty}^{\infty} f(x, y) d x d y, m_{0,1}=\int_{-\infty}^{\infty} \int_{-\infty}^{\infty} y f(x, y) d x d y
\end{aligned}
$$

For digital power network lines' image $f(x, y)$, use the form of double sum in place of integral, the $(p+q)$-order moment of point $(x, y)$ can be obtained as $m_{p, q}=\sum \sum x^{p} y^{q} f(x, y)$, zero order moment $m_{0,0}=\sum \sum f(x, y), m_{0,0}$ is used to describe the number of points in this region.

The first order moment of $f(x, y)$ can be described as:

$$
m_{1,0}=\sum_{x} \sum_{y} x f(x, y), m_{0,1}=\sum_{x} \sum_{y} y f(x, y)
$$

The central moment of $f(x, y)$ can be described as:

$\mu_{p, q}=\sum \sum\left(x-x_{c}\right)^{p}\left(y-y_{c}\right)^{q} f(x, y)$

$\mu_{p, q}$ is an invariant under translation and scaling transformation.

(3) Image statistical feature. A two-dimensional array is a simple way to describe the image area. The information of all pixels in the image matrix can be obtained through the pixels' coordinates, to get corresponding image information from matrix. So a person may carry on the statistical analysis of a image's gray value, and use it as the statistical characteristics of the image:

The mean value is:

$\mu=\sum_{i=0}^{N-1} i p(i)$

The variance is:

$$
\sigma_{i}^{2}=\sum_{i=0}^{N-1}(i-\mu)^{2} p(i)
$$

The skewness is:

$$
i_{k}={\frac{1}{\sigma_{i}^{3}}}_{i=0}^{N-1}(i-\mu)^{3} p(i)
$$

The value of kurtosis is :

$$
i_{k f}={\frac{1}{\sigma_{i}^{4}}}_{i=0}^{N-1}(i-\mu) p(i)-3
$$

Energy is:

$$
i_{n}={ }_{i=0}^{N-1}[p(i)]^{2}
$$

The generalized entropy is:

$$
i_{k}=-\sum_{i=0}^{N-1} p(i) \log [p(i)]
$$

Of which, $\mathrm{N}$ is used to describe the total count of gray-scale; $p(i)$ is used to describe the gray-scale probability density of the power network lines' image.

Image features' classification and matching based on SVM. Support vector machine (SVM) has a great advantage in tackling small sample, nonlinear and high dimensional pattern recognition 
problem. After training samples to complete learning according to the known category, one can obtain optimal hyperplane among sub images of different categories within the same area. Then conduct learning classification on all the test samples, obtain the correct classification rate $\left[R_{A_{11}}, R_{A_{12}}, \cdots, R_{A_{77}}\right]$ of sub images at different locations, of which, $A_{i j}$ is used to describe the sub image at the position $(i, j)$ after the test sample is divided, $R_{A_{i j}}$ is used to describe the accuracy of classification of sub images of all test samples through SVM.

The values of the classification accuracy reflect the degree of differentiation of the sub images of all samples in electric network line image library, so, the weight of each sub image when matching is different, namely:

$$
\left[\lambda_{A_{1}}, \lambda_{A_{2}}, \cdots, \lambda_{A_{7}}\right]=\frac{1}{\sum_{i=1}^{7} \sum_{j=1}^{7} R_{i j}}\left[R_{A_{1}}, R_{A_{2}}, \cdots, R_{A_{7}}\right]
$$

Of which, $\lambda_{i j}$ is used to describe weights of sub images in the process of matching. After obtaining the weight of each image in matching, conduct fusion on the classification results of all the sub image of the entire to-be-matched vein images, judge whether the image coincides with the power network line breakage characteristics, so as to realize the identification of power lines' damage:

$$
\begin{aligned}
& C_{p}=\sum_{A_{i j} \in I} \lambda_{A_{i j}} \\
& C_{u}=\max \left\{C_{1}, C_{2}, \cdots, C_{m}\right\}
\end{aligned}
$$

Of which, use $I$ to describe the $p$ th kind of power network lines' image; $C_{p}$ is used to describe the possibility of the to-be-matched power network line image belongs to the broken line image; $C_{u}$ is used to describe to-be-matched image is most likely to belong to the broken line image. Set a reasonable threshold $T$, if $C_{u}>T$, to-be-matched image is broken power network line image; otherwise, to-be-matched image is not broken power network line image.

\section{Simulation results}

In order to validate the proposed power network line damage identification method based on image feature analysis is effective, relevant experimental analysis is required. The simulation experiment is under the environment of Matlab 7.0. Respectively using the proposed method and the traditional method based on wavelet neural network for the breakage identification of broken lines of the five samples of power network lines, obtained results are shown in Table 1.

Table 1 Comparison results of identification accuracy of the proposed method and the traditional method

\begin{tabular}{|l|l|l|l|l|}
\hline $\begin{array}{l}\text { Number of } \\
\text { samples }\end{array}$ & $\begin{array}{l}\text { Training } \\
\text { samples }\end{array}$ & $\begin{array}{l}\text { Test } \\
\text { samples }\end{array}$ & $\begin{array}{l}\text { Identification accuracy of } \\
\text { the proposed method }\end{array}$ & $\begin{array}{l}\text { Identification accuracy of } \\
\text { traditional method }\end{array}$ \\
\hline 1 & 50 & 500 & 0.95 & 0.82 \\
\hline 2 & 50 & 500 & 0.92 & 0.84 \\
\hline 3 & 50 & 800 & 0.89 & 0.75 \\
\hline 4 & 50 & 800 & 0.94 & 0.78 \\
\hline 5 & 50 & 800 & 0.91 & 0.73 \\
\hline
\end{tabular}

Analyzing Table 1, it can be seen that the accuracy to identify power network line damage using the proposed method is much higher than that using the traditional method based on wavelet neural network, this is because the method in this paper, first of all, extracts the characteristics of the broken lines, takes into account the various line broken situations into full play, so the accuracy is higher.

To further verify the validity of the method in this paper, on the basis of the above experiments, count the time for identification using the method of this paper and the traditional method based on wavelet neural network, the results are shown in Figure 1. 


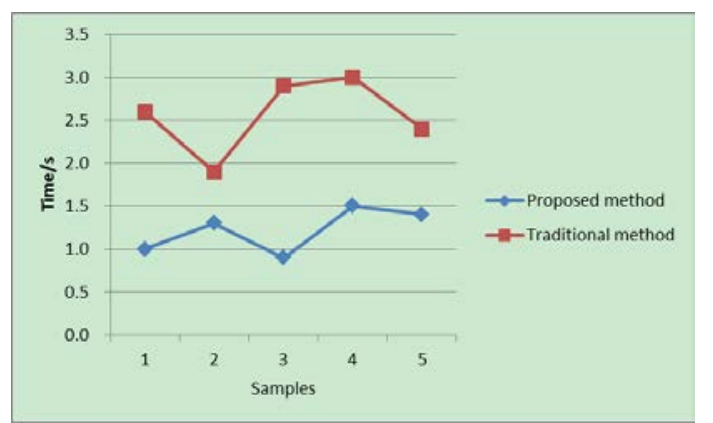

Figure1 Comparing results of efficiency of the proposed method and the traditional method

Analyzing Figure 1, it can be seen that, to identify the same sample, the time needed for the proposed method is far less than that needed for the traditional method based on wavelet neural network, which shows that this method not only has high identification accuracy, but also has high identification efficiency.

\section{Conclusion}

This paper put forward a kind of power network line damage identification method based on image feature analysis, the initial processing is done on the power network line damage image through the contour features. Power network line breakage characteristics is further described through torque and outline description. For digital electric power network lines' images, the form of the double sum replaces integrals, statistical analysis is done on the image's gray value, which is used as the statistical characteristics of the image. After training samples to finish learning according to the known category, all the test samples go through learning classification, the correct classification rate of the sub images at different positions is obtained, the weight of each image when matching is calculated. Conduct fusion on the classification results of all the sub images of all to-be-matched vein images, judge whether the image matches with power network line breakage characteristics, so as to realize damage identification of the power lines. The simulation results show that the proposed method has high identification accuracy.

\section{Reference}

[1] SANG Qing-bing, SU Yuan-yuan, LI Chao-feng, WU Xiao-jun*, No-reference blur image quality assemssment based on gradient similarity, Journal of Optoelectronics.Laser, 2013, 24(3):573-577

[2] MI Zeng-zhen, XIE Zhi-jiang, CHEN Tao, CHU Hong-yu, FAN Bing, Key technology of image enhancement and edge extraction for heavy rail, Optics and Precision Engineering, 2012, $20(7): 1645-1652$

[3] HAN Xi-zhen, ZHAO Jian, Enhancement of image texture and contrast combined with partial differential equation, Optics and Precision Engineering, 2012, 20 (6) : 1382-1388

[4] YU Hai-liang, WANG Yue-xin, ZHANG Jin, YANG Shuang-xi, LIU Xiang-hua, Review on factors of surface cracks in slab and their behavior during rolling, Forging \& Stamping Technology, 2010, 35 (2) : 1-5

[5] Ouyang Qi, Zhang Min, Zhao Liming, Zhang Lizhi, Study on Continuous Casting Billet Surface Defect Detection Based on Pulsed Eddy Current Technique, China Mechanical Engineering, 2010 (10) : 1235-1239 